\title{
EL USO DE LAS PRODUCCIONES ESCRITAS O VISUALES DEL CONSULTANTE COMO INSUMO TÉCNICO EN LA PSICOTERAPIA DINÁMICA ${ }^{1}$
}

\section{The Use of the Written or Visual Productions of Patient as a Technical Input in Psychodynamic Psychotherapy}

\author{
Gabriel Jaime Garzón Ruiz ${ }^{2}$
}

Recibido: 2018-03-30 Aceptado: 2018-10-12

\begin{abstract}
Resumen: El arte y las actividades creativas han ido abriéndose un campo importante en el área de la clínica psicológica debido a que, por medio de estas expresiones, el sujeto puede proyectar y tramitar tensiones internas —que se le dificulta simbolizar a través del uso de la palabra-, así como construir nuevas formas de sentido subjetivo. Durante la investigación se realizó una inmersión en el uso que los psicoterapeutas dinámicos le dan a las producciones gráficas y escritas de sus consultantes en el espacio terapéutico con el objetivo de elucidar los objetivos de evaluación e intervención referentes al uso de estas producciones y cuestiones acerca de la técnica.
\end{abstract}

Palabras clave: terapia psicoanalítica, relaciones objetales, pintura, escritura, actividades creativas.

\begin{abstract}
The arts and the creative activities have been acquiring a place of relevance in the area of psychotherapy because it has been found that, through these expressions, the subject can project and process internal tensions that it is difficult for him to symbolize through the spoken language, as well as to build new forms of subjective meaning. The present research report makes an immersion in the use that the psychodynamic psychotherapists give to the graphic and written productions made by their patients. The research aim was to elucidate issues in relation to the technique and the establishment of clinical objectives as psychological evaluation and intervention.
\end{abstract}

Keywords: psychoanalytic psychotherapy, object relations, painting, writing, creative activities.
Para citar este artículo en APA: Garzon, G. (2018). El uso de las producciones escritas o visuales del consultante como insumo técnico en la psicoterapia dinámica. Revista de Psicología Universidad de Antioquia, 10(2), 103-129. DOI: 10.17533/udea.rp.v10n2a05
Artículo de investigación derivado de la Maestría en Psicología Clínica. Universidad de San Buenaventura, Medellín, Colombia.

2. Magíster en Psicología Clínica. Psicólogo clínico Viva 1A IPS. Correo: gabrielgarzon_85@hotmail.com; https:// orcid.org/0000-0002-9341-9008. 


\section{Introducción}

Tradicionalmente en el espacio de la psicoterapia dinámica el principal medio e insumo con el que cuenta el terapeuta es el relato histórico y experiencial del consultante. Sin embargo, se ha encontrado en un sondeo realizado a psicoterapeutas dinámicos — docentes de la Universidad San Buenaventura, sede Medellín-, que las producciones escritas y visuales de los pacientes, tales como poemas, dibujos, pinturas, composiciones musicales, etc., también juegan un papel de vital importancia para el flujo del proceso psicoterapéutico.

Si bien en la literatura psicoanalítica se pueden encontrar reflexiones teóricas acerca de los procesos inconscientes y psicodinámicos que intervienen en el acto creativo y artístico, como puede verse principalmente en autores clásicos como Sigmund Freud y Donald Winnicott (1995), también se evidencia la poca información acerca del uso clínico que les han dado los psicoterapeutas dinámicos a tales producciones. En este sentido, el presente artículo tiene como propósito presentar, comprender y organizar el uso que los psicoterapeutas dinámicos le dan a las producciones visuales y escritas realizadas por los consultantes en el espacio clínico.

Para la consecución de dicho objetivo se realizaron entrevistas semiestructuradas a cinco psicólogos dinámicos que han utilizado dichas producciones como insumo facilitador del proceso terapéutico. El texto se encuentra estructurado en cinco partes: 1) conceptualización de las producciones escritas y visuales, perspectivas psicoanalíticas y antecedentes; 2) metodología; 3) resultados; 4) discusión; y 5) conclusiones, limitaciones y sugerencias.

\section{Acerca del concepto de producciones escritas/visuales}

- PEV-

En el presente texto las PEV están directamente vinculadas con el arte, ya que son productos simbólicos que nacen a partir de la creatividad de cada sujeto. Mediante dichas producciones se comunican deseos, pensamientos y sentimientos que tienen los consultantes en relación con sí mismos, los otros 
y el mundo (Hallar, 2015, p. 137). En este orden de ideas, para Hallar (2015) el arte hace referencia al «resultado de un ejercicio creativo intencionado que, mediante el uso de técnicas y herramientas concretas, genera un producto de una modalidad perceptiva particular» (p. 132).

Es importante resaltar que para este texto se hace caso omiso de una concepción de arte profesionalizado en el que se requiere o exige una rigurosidad en la técnica. Ahora bien, las PEV deben tener una característica esencial y es que el consultante, como sujeto creador de éstas, le atribuya un valor, una significación, un sentido subjetivo o una carga representacional-afectiva a nivel consciente o inconsciente, lo cual debe ser objeto de análisis para el psicoterapeuta.

\section{Arte y psicoanálisis}

Teniendo en cuenta la conexión entre las PEv y el arte, el siguiente apartado tiene como objetivo exponer los referentes teóricos que presenta el psicoanálisis acerca de los procesos inconscientes y psicodinámicos que se dan en el acto de la creación artística y la creatividad, para así brindarle un sustento teórico a esta investigación.

\section{Teoría freudiana del arte}

En el texto Un recuerdo infantil de Leonardo Da Vinci, Freud (1986a) pretende realizar una reconstrucción de la historia infantil de Da Vinci y determinar ciertos rasgos estructurales de su personalidad a partir de la triangulación entre el conocimiento de sus biografías, lo que se puede llegar a analizar en sus creaciones artísticas y sus propias construcciones de la teoría psicoanalítica. Es así como Freud (1986a) busca aproximarse a los conflictos inconscientes que Da Vinci plasmaba en su obra; esto es posible, según el autor, porque «el artista expresa mediante creaciones sus mociones anímicas, escondidas para él mismo» (p. 100), lo que se puede asociar al mecanismo de proyección.

Para ser más específicos, Laplanche y Pontalis (1996) sostienen que en la proyección «el sujeto percibe el medio ambiente y responde al mismo en fun- 
ción de sus propios intereses, aptitudes, hábitos, estados afectivos duraderos o momentáneos, esperanzas, deseos, etc.» (p. 307). Es por esto por lo que sería importante establecer una diferencia entre: (i) el mecanismo de proyección normal en todos los seres humanos, como es descrito anteriormente; y (ii) la proyección como mecanismo de estructuras paranoides, lo cual no tiene cabida en el presente reporte de investigación.

De manera análoga es menester señalar que el creador en su producción suele plasmar sus contenidos inconscientes y realizar una descarga pulsional. En el caso de Da Vinci, Freud (1986a) arguye que a través de sus creaciones el pintor logra tramitar en cierta medida la tensión que provocaba la represión de su homosexualidad (p. 75), así como el amor incestuoso que mantenía hacia su madre (1986a, p. 110). Esto es lo que se conoce como mecanismo de sublimación, sobre el cual Freud (1986a) resalta que:

La observación de la vida cotidiana de los seres humanos nos muestra que la mayoría consigue guiar hacia su actividad profesional porciones muy considerables de sus fuerzas pulsionales sexuales. Y la pulsión sexual es particularmente idónea para prestar esas contribuciones pues está dotada de la aptitud para la sublimación; o sea que es capaz de permutar sus metas inmediatas por otras, que pueden ser más estimadas y no sexuales (p. 72).

Dicho lo anterior, la sublimación también se ve directamente asociada a la fantasía (Freud, 1986a), ya que por medio de ella el sujeto puede cambiar algo de la realidad que lo limita para el cumplimiento de sus deseos; así pues, la fantasía constituye un medio para la consecución del placer.

En este mismo sentido, Freud en su obra literaria Dostoyevski y el parricidio (1986b) arroja de nuevo la hipótesis de la creación artística como evidencia material de conflictos inconscientes infantiles. En este texto el autor concluye que la clase de personajes elegidos por Dostoyevski para la creación de sus libros -criminales, ludópatas, etc.- no es otra cosa que la representación simbólica del interior del escritor (1986b, p. 176).

A su vez, otro aporte de Freud (1986c) a la comprensión del fenómeno de la creación del artista se puede encontrar en su texto El Moisés de Miguel Ángel, escrito en el que el Freud intenta interpretar la escultura de Moisés, atribuyéndole a Miguel Ángel una intencionalidad consciente o inconsciente que sub- 
yace en la producción del artista. Ahora, si bien se puede observar en el texto de Da Vinci una focalización de Freud (1986a) sobre la obra artística como reflejo de conflictos y un resultado del mecanismo sublimatorio, en el análisis del Moisés de Miguel Ángel la tesis freudiana se concentra en señalar que tras la creación hay una motivación susceptible a la interpretación (Freud,1986c).

\section{Teoría winnicottiana de la creatividad}

Uno de los grandes aportes teóricos de Winnicott (1995) al psicoanálisis consiste en el concepto de fenómenos transicionales, entre los cuales se encuentran el objeto transicional y el espacio transicional. En Realidad y juego, Winnicott dice:

Introduzco los términos «objetos transicionales» y «fenómenos transicionales» para designar la zona intermedia de experiencia [...] entre el erotismo oral y la verdadera relación de objeto, entre la actividad creadora primaria y la proyección de lo que se ha introyectado (1995, p. 18).

Agrega además que el espacio transicional «constituye la mayor parte de experiencia del bebé, y se conserva a lo largo de la vida en las intensas experiencias que corresponden a las artes y la religión, a la vida imaginativa y a la labor científica creadora» (Winnicott, 1995, p. 32). Es de esta manera como Winnicott (1995) incluye el fenómeno de la creatividad en esta zona intermedia de experiencia; una zona que no es ni interior, ni es exterior, es ambas, rompiendo así con la dualidad clásica dada entre la realidad objetiva y la realidad subjetiva.

Siguiendo la línea de los fenómenos transicionales, otro concepto fundamental en Winnicott (1995) es el de objeto transicional, el cual Laplanche y Pontalis (1996) definen como:

Objeto material que posee un valor electivo para el lactante y el niño pequeño, especialmente en el momento de dormirse (por ejemplo, un ángulo del cubrecama, una toalla que chupetea). El recurrir a objetos de este tipo constituye, según el autor, un fenómeno normal que permite al niño efectuar la transición entre la primera relación oral con la madre y la verdadera relación de objetos (p. 265). 
Por su parte, Sonia Abadi (1996) dice que el objeto transicional «recibe tanto el afecto tranquilo, como excitación amorosa y hostil. Tendrá que sobrevivir al amor y al odio» (p. 36); además considera que el objeto transicional «representa el pecho de la madre como el pecho internalizado» (p. 37). Por otro lado, en relación con el juego, la autora asegura que éste «cumple una función esencial en el manejo de la agresión y la destructividad [...] el objeto puede ser dańado o destruido y luego reparado, ensuciado y vuelto a limpiar, matado y revivido» (Abadi, 1996, p. 52). De suerte que el juego facilita en el niño el proceso de integración de afectos ambivalentes, promoviendo de esta forma una superación del mecanismo de disociación -objetos buenos y malos-.

\section{Antecedentes}

En Arte, locura y psicoterapia, Rodrigo Hallar (2015) -quien centra su libro en la aplicación del arte en el trabajo con pacientes de estructuras psicóticas- explica que tanto el acto creativo como su contemplación le brinda una herramienta al individuo para que se relacione más estrechamente con su mundo interno y potencializa su capacidad reflexiva respecto a las relaciones con los otros y con el mundo (p. 137).

Llegados a este punto, en relación con la escritura, Adorna y Covarsí (2016) sugieren que escribir «ayuda a que las conexiones entre los dos hemisferios cerebrales se realicen con mayor eficacia, puesto que actúan unidas la parte racional y la emocional y creativa» (p. 13). Asimismo, Seagel, como se cita en Adorna y Covarsí (2016), afirma con base en estudios neurocientíficos que «el simple hecho de anotar la descripción de una experiencia difícil, puede reducir la reactividad fisiológica y aumentar la sensación de bienestar, aunque lo que se haya escrito no lo enseñemos a nadie» (p. 14).

Conforme a lo anterior, en la psicoterapia se ha trabajado con dos tipos de producciones escritas: las autobiográficas y la poesía. Sobre las producciones autobiográficas, Martín (2015) argumenta que es una vía por la cual se puede conocer mejor al paciente, reemplazando la tradicional anamnesis clínica (p. 
2). En igual medida, respecto a los aspectos que se pueden recolectar con la actividad autobiográfica, García y Martínez, como se cita en Martín (2015), sostienen que se pueden hallar:

1) Las personas que son relevantes para el cliente y cuál es el papel que desempeñan en su vida. 2) Las metas y objetivos del cliente, que aparecen fundamentalmente en el desafío vital, la tarea vital y la descripción de los futuros alternativos. 3) Información sobre qué contenidos regulan la visión del mundo del sujeto. 4) Las estrategias de afrontamiento que lleva a cabo y los recursos de los que dispone. 5) Los moduladores y limitadores vitales que ha tenido el cliente y que le han llevado a tomar decisiones en uno u otro sentido. 6) El contenido subjetivo de su experiencia personal, es decir, su punto de vista sobre lo que ha pasado y cómo se ha desarrollado su vivencia (p. 2).

En esta misma vía, Lanza (2006) afirma que el acto de escribir autobiográficamente le permite al sujeto tomar distancia de su propia experiencia, analizarla más racionalmente y desde otros puntos de vista, lo que implica, a su vez, que tome consciencia de su propia reacción afectiva y se mitigue el nivel de afectación emocional (p. 168).

En cuanto a la poesía, de la mano de Shafi (2010) se puede decir que es un «lenguaje métrico y significativo que usa el ritmo, el sonido, la imagen y especialmente la metáfora en un patrón estructural que invoca emociones» (p. 87). Más aún, es una herramienta clínica que, aplicada a la terapia, ayuda a desarrollar la creatividad, los significados personales y un puente para que el consultante pueda llegar al insight (2010, p. 88).

Como lo sugiere Shafi (2010), es relevante resaltar el proceso que se presenta dentro de la metáfora, ya que ésta tiene el potencial de establecer una dialéctica entre una imagen y un concepto. En resumen, la poesía se convierte en un producto en el que, paradójicamente, juegan elementos de lógica verbal para evocar imágenes que le posibilitan al sujeto acceder a una dimensión no verbal de la experiencia.

En continuidad con este tipo de producción, Hallar (2015) afirma que el poeta es capaz de manipular el lenguaje, de controlarlo y administrarlo, y, debido a la relevancia que tiene el lenguaje en la construcción del psiquismo, «el escribir poesía puede llegar a ser un significativo ejercicio de flexibiliza- 
ción emocional y cognitiva» (p. 154). Igualmente, para este autor la poesía se convierte en un medio para comunicar y compartir con los otros experiencias íntimas de compleja significación en un lenguaje ordinario (2015, p. 155).

Por otra parte, en cuanto a las expresiones de carácter visual, el dibujo o la pintura, se establece como núcleo central la imagen. Representantes de la psicología cognitiva como Guidano, como se cita en Hallar (2015), han expuesto que la imagen brinda información de la organización psíquica, tanto a un nivel de almacenamiento de información relativa a la constitución mental, como de jerarquización de la experiencia y desarrollo biográfico (p. 175).

Así, por ejemplo, al trabajar por medio de la pintura con pacientes con trastornos mentales severos, Pérez (2012) registra que «la pintura llegó a ser una forma de expresión que llevó a los pacientes a estadios muy tempranos del desarrollo de la personalidad, a estadios de las relaciones objetales tempranas» (p. 146). También afirma que los pacientes con los que trabajó lograron por medio del dibujo reducir la ansiedad, establecer mejores vínculos con el terapeuta y podían lograr narraciones con mayor contenido asociativo de su mundo interno (2012, p. 147).

En este mismo sentido, Dumas y Aranguren (2013) argumentan que las producciones gráficas «proveen la capacidad de conectarse con los colores y las formas que están en estrecha relación con el universo afectivo» (p. 43); de modo que en este proceso los afectos que están reprimidos transmutan en energía constructiva y generan una tendencia a integrar representaciones y afectos disociados.

De manera general, Marinovic, como se cita en Morales (2008), destaca que practicar actividades artísticas: (i) enriquece la percepción; (ii) amplía la imaginación y la creatividad; (iii) facilita el orden y la organización; (iv) permite la flexibilidad del pensamiento; (v) aumenta la capacidad de autorreflexión; (vi) construye un puente entre realidad subjetiva y objetiva; (vii) provee un medio esencial para la sublimación; (viii) produce catarsis; (ix) permite el despliegue de las capacidades del sujeto y la consecución de nuevas destrezas; y (x) enriquece el valor de sí mismo, el autoconcepto, la autoeficacia.

Para terminar, según García (2018), Lacan brinda su aporte principal sobre la comprensión del arte relacionándolo con lo bello: 
[...] el cruce entre lo bello y el deseo parece darse en el margen de dolor, pero no del dolor del masoquismo que, de acuerdo con Lacan, es un bien que se intercambia, sino de lo bello con su crueldad y sincronía. Parecería entonces que lo bello en tanto dolor estuviera preso de una pasión que irrumpe y descompone la armonía supuesta del cuerpo especular que le otorga su imagen vista en el espejo (pp. 61-62).

Lo anterior significa que, de acuerdo con Lacan, en lo bello el sujeto realiza una ruptura con la imagen especular con la que se identifica y en la que se aliena a sí mismo en las demandas y los significantes que le han sido impuestos por el Otro. Dicha renuncia implica como consecuencia un dolor, sin embargo es un dolor no masoquista, un dolor que no tiene su origen en los castigos y el sadismo del superyó, sino un dolor que viene de una renuncia a la imagen narcisista en la cual se halla preso; un duelo que subyace a su proceso de autonomía y liberación del Otro.

\section{Metodología}

La presente investigación se realizó desde un enfoque cualitativo, el cual tiene como objetivo obtener una comprensión profunda de los significados y sentidos de la situación estudiada, tal y como nos la presentan las personas. El enfoque cualitativo se caracteriza por la flexibilidad del diseño de investigación y la emergencia de categorías a lo largo del proceso investigativo (Salgado, 2007). Igualmente, se ha utilizado un método hermenéutico-interpretativo bajo una estrategia de estudio de caso; así, se eligió en primer lugar una escuela teórica a partir de la cual se buscó dar cuenta del fenómeno abordado de forma empírica (Ángel, 2011).

Teniendo en cuenta lo anterior, dicho estudio de caso de práctica clínica tuvo como horizonte la comprensión del saber y el quehacer que han construido los psicoterapeutas que han puesto las producciones escritas y visuales de sus consultantes al servicio del espacio terapéutico. De ese modo, los criterios de elección de los cinco terapeutas entrevistados fueron: 1) que fueran psicoterapeutas de orientación psicodinámica; 2) que tuvieran experiencia clínica 
superior a los diez años; 3) que hubieran utilizado las producciones escritas y visuales de sus consultantes al servicio del trabajo terapéutico.

Los sujetos entrevistados se seleccionaron a partir de un muestreo cualitativo tipo bola de nieve, en el que cada terapeuta entrevistado remitía por recomendación de conocimiento a otro terapeuta (Salamanca y Martín-Crespo, 2007). La estrategia utilizada para generar la información fue, en un primer momento, una entrevista semiestructurada de exploración, construida con base en categorías extraídas de una primera revisión de la teoría psicoanalítica en relación con el arte.

Posterior a esto se realizó un análisis de datos generados en ATLAS.ti usado «para el análisis cualitativo de datos» (ATLAs.ti, 2018, p. 1)—, análisis desde el cual emergieron nuevas categorías y subcategorías. A partir de ellas se construyó una segunda y última entrevista semiestructurada, aplicada a los mismos psicoterapeutas con el propósito de complementar la información inicial y refinar la construcción de las categorías que surgieron a lo largo del proceso investigativo.

Finalmente se realizó la triangulación de la información de las categorías iniciales y emergentes con las categorías teóricas provenientes de la literatura psicodinámica sobre el tema, dando estructura a los resultados que se presentarán a continuación.

\section{Condiciones éticas de la investigación}

Este proyecto de investigación fue aprobado por el Comité de Bioética de La Universidad de San Buenaventura según la Resolución nº 8430 de 1993 del Ministerio de Salud de Colombia. Se tuvo en cuenta el Código Deontológico y Bioético del psicólogo y la firma del consentimiento informado, respetando el derecho a la intimidad al omitir cualquier información como nombres, lugares y fechas que permitieran identificar a los sujetos de estudio, esto con el fin de salvaguardar la identidad de los participantes. 


\section{Resultados}

Con el objetivo de realizar una primera aproximación a una organización «esquemática» respecto al uso de las producciones escritas y visuales de los consultantes como instrumento técnico en la psicoterapia dinámica, la presente sección se dividirá en dos partes, a saber: (i) los objetivos de la evaluación e intervención y (ii) las cuestiones acerca de la técnica.

Objetivos de evaluación e intervención respecto al uso de las PEV

\section{Promover la asociación libre}

Para mi lo más importante es coger esta producción estética del consultante como objeto que lo estimule para asociar libremente (entrevistado, comunicación personal, noviembre de 2017).

La asociación libre, significada como un «método que consiste en expresar sin discriminación todos los pensamientos que vienen a la mente, ya sea a partir de un elemento dado [...], ya sea de forma espontánea» (Laplanche y Pontalis, 1996, p. 35), es el objetivo al que más apuntan los psicoterapeutas durante las entrevistas. Según la información generada, hay dos formas de ver la PEV como asociación libre: (i) la creación en sí misma, por lo que la PEV debe considerarse como un resultado de la asociación libre, y (ii) el discurso del paciente que puede desplegarse a partir de su PEV como estímulo.

En caso de que el terapeuta utilice la PEV como estímulo para promover el discurso del paciente —uso más resaltado por los terapeutas-, las producciones escritas y las visuales se diferencian de acuerdo con su abordaje. En caso de que el consultante haya utilizado una producción de tipo visual, los psicoterapeutas le piden al paciente de forma explícita: «Hábleme de eso», con el fin de hacer emerger el discurso subjetivo del paciente, que narre su creación, porque al hablar de ésta estará hablando de sí mismo: «Algunos pacientes al 
traer sus escritos me piden que yo los lea, pero para mí es importante que él sea quien lo lea, porque en la entonación se transmite también información» (entrevistado, comunicación personal, noviembre de 2017).

Es por esto por lo que, respecto a las producciones escritas como autobiografías y poesías, hay una tendencia de los psicoterapeutas de pedirle al sujeto que lea su propia producción bajo el propósito de atender los ritmos, tonalidades, intensidades, manifestaciones conductuales y actos fallidos que pueda tener el consultante en los distintos momentos de la lectura de su PEV.

\section{Identificación de conflictos}

\section{A través del poema que me trajo la consultante a esa sesión pu- dimos llegar al tema del padre, que nunca habiamos abordado en el proceso, y seguir trabajando sobre él (entrevistado, comunicación personal, noviembre de 2017).}

En relación con la anterior subcategoría se ha encontrado durante la investigación que a través del uso de las PEv los psicoterapeutas han podido identificar conflictos inconscientes que hasta el momento no habían podido emerger en el espacio clínico —o que al menos ellos no habían podido identificar a través del discurso del consultante-, así como confirmar hipótesis de conflictos que el terapeuta había podido identificar con algunos indicios anteriores.

En este punto se puede evidenciar la importancia del aporte freudiano respecto a sus concepciones de las creaciones artísticas, especialmente su teoría acerca de la proyección, en la que constantemente percibimos el mundo exterior desde nuestro mundo interno (Laplanche y Pontalis, 1996, p. 307). Es necesario recalcar que para Freud (1986a) la obra de arte es un resultado material de conflictos y deseos inconscientes (p. 100), teoría que soporta el uso de las pruebas proyectivas (Laplanche y Pontalis, 1996, p. 307). 


\section{Objeto transicional — contención de ansiedad-}

En la presente subcategoría es vital traer a colación el concepto creado por Winnicott (1995) referente al objeto transicional. Stevenson, como se cita en Mesones (2014), sostiene que una de las funciones que tiene este objeto es «su utilidad para lidiar en situaciones de ansiedad, inclusive en etapas más allá de la niñez» (p. 11). Durante el análisis de la información surgieron dos formas en las que el uso de las PEv sirvió como objeto transicional:

a) Herramienta usada por el mismo consultante entre sesiones para contener sus niveles de ansiedad, utilizando el acto creativo de escritura o de dibujo para plasmar allí y desahogar emociones y pensamientos que eran registrados para transmitir en la próxima sesión a su psicoterapeuta: «Ella escribía para mí, y eso la ayudaba como a contenerse mientras llegaba el momento de la sesión» (entrevistado, comunicación personal, noviembre de 2017).

b) El uso de las PEV del consultante como forma de ser recordado por la figura del terapeuta. En este caso, se presenta comúnmente con sujetos institucionalizados, en su mayoría niños o adolescentes, que tras haber vivido experiencias de abandono reviven esta misma experiencia traumática ante el psicoterapeuta, por lo que han encontrado en varios casos una necesidad de «regalar» sus PEV a su psicólogo con la intención de contener su angustia de ser olvidados y mantener un vínculo objetal, utilizando un objeto mediador que posibilite el recuerdo, en este caso, su propia creación.

En síntesis, en este tipo de casos el objeto cumple una función de contener la angustia de separación al igual que el objeto transicional winnicottiano, sólo que de forma inversa, pues es el otro quien queda al resguardo del objeto: «Esta niña, que tenía un miedo a que se olvidaran de ella, me regala sus dibujos y me dice: "Vea, para que nunca se olvide de mí", y es el recurso que tiene ella para afrontar su angustia» (entrevistado, comunicación personal, noviembre de 2017). 
Vencimiento de resistencias y defensas

Me serví de la creación del paciente como una forma de pretexto para dar lugar a la emergencia de la palabra, ya que hay personas que se les dificulta mucho hablar de su intimidad (entrevistado, comunicación personal, noviembre de 2017).

Éste ha sido el objetivo que se han propuesto los psicoterapeutas, en la mayoría de los casos con niños, razón por la cual actividades de producción visual como el dibujo son utilizadas con esta población "pensando en la dificultad del lenguaje y del incompleto desarrollo del pensamiento» (Pérez, 2012, p. 146). Sin embargo, la etapa evolutiva no es el único factor que dificulta el tratamiento hecho mediante el uso de la palabra, por lo que las PEV se han empleado también en el trabajo psicoterapéutico con adultos a los cuales se les dificulta la expresión verbal de sus experiencias, pensamientos y emociones.

Se debe agregar que esta dificultad puede presentarse por una activación de mecanismos defensivos, fenómeno entendido por Coderch (1990) como un conjunto de "procesos intrapsíquicos con que el yo intenta protegerse del peligro del dolor mental, y a su manifestación durante el tratamiento lo denominamos resistencias» (p. 130). Un claro ejemplo de dichos mecanismos es la denominada evitación, en la cual la persona evita hablar o enfrentar el estímulo o situación desencadenante de ansiedad (1990, p. 158).

Por consiguiente, el uso de las PEv en este caso ha posibilitado que el proceso fluya de una mejor manera en momentos de estancamiento en el proceso clínico, puesto que en dichas creaciones hay una menor posibilidad de que el sujeto active mecanismos de defensa, permitiendo que emerjan nuevos elementos que hubieran sido más complejos de evidenciar o de traer a colación con el uso de la entrevista clínica tradicional. 


\section{Pago simbólico}

A propósito del escrito que era un poema, este sujeto se compromete a seguir llevando escritos cada sesión como pago de la atención, y en este caso particular ese pago es el que le permite al sujeto engancharse al proceso (entrevistado, comunicación personal, noviembre de 2017).

Por último, se registró en la investigación el uso de las PeV como forma de pago simbólico, es decir, las PEV de los consultantes eran usadas como medio de pago al psicoterapeuta cuando éstos presentaban problemas económicos para pagar las citas, ya sea de fundaciones, instituciones de salud como las EPS o, incluso, de la clínica particular.

$\mathrm{Al}$ igual que en las situaciones anteriormente expuestas, el pago simbólico con las PEV también ha sido un objetivo delimitado por los psicoterapeutas en el espacio clínico psicológico con niños y adolescentes debido a que estos procesos son normalmente pagados por sus padres y no por ellos.

Al respecto dice Freud (1986d):

[...] muchas de las resistencias del neurótico se acrecientan enormemente por el tratamiento gratuito; en la mujer joven, la tentación contenida en el vínculo transferencial, en el hombre joven, su renuencia al deber del agradecimiento, renuencia que proviene del complejo paterno (pp. 133-134).

Así las cosas, se ha encontrado en la investigación que el uso de dicho pago simbólico ha servido en varios casos para que los consultantes se comprometan en mayor medida con el espacio psicoterapéutico, asignándole un valor que no le era atribuido cuando no pagaban nada por él. De este modo, la aceptación del pago simbólico por parte del terapeuta promueve una valoración del self del consultante, lo que refuerza la autoestima del último, ayudando también al establecimiento del vínculo terapéutico. 


\section{Cuestiones acerca de la técnica}

\section{Técnicas verbales asociadas}

— Interrogar al paciente: para Fiorini (1984) interrogar al paciente consiste en «pedirle datos precisos, ampliaciones y aclaraciones del relato y explorar en detalle sus respuestas» (p. 141). Respecto a dicha intervención verbal se encontró en la investigación que los terapeutas interrogan detalladamente al paciente acerca de su PEv. Algunas de las preguntas más frecuentes han sido: «¿En qué momento lo hizo? ¿Por qué lo trae a consulta? ¿Por qué lo trae a esa sesión en particular? ¿Qué estaba sintiendo cuando lo hizo? ¿Qué significa para usted? ¿Qué quería expresar ahí?» (entrevistados, comunicación personal, noviembre de 2017).

— Señalamientos: Fiorini (1984) describe esta técnica como la acción de «señalar relaciones entre datos, secuencias, constelaciones significativas» (p. 142). Como ejemplos retomados de la casuística expuesta por los psicoterapeutas entrevistados, se presenta el siguiente señalamiento: «¿Se da cuenta que sus poemas siempre son alusivos a su padre?, pero usted aquí en consulta nunca ha querido hablar de él» (entrevistados, comunicación personal, noviembre de 2017).

— Interpretación: para Coderch (2012):

El acto interpretativo es aquel mediante el cual el analista intenta brindar al paciente la posibilidad de tener un conocimiento acerca de su inconsciente y de sus dinamismos mentales en general, conocimiento al que en el lenguaje psicoanalítico llamamos insight (p. 320).

Para este caso, la interpretación recaería sobre su PEV o lo que el sujeto hable acerca de ella. Durante la investigación se presenta un consenso entre los psicoterapeutas respecto a que la interpretación del uso de las PEV se ha de dar en forma de construcción dialéctica. Esto quiere decir que la interpretación del psicoterapeuta no se hace de una manera rígida ni unilateral, sino que el sentido de la PEV se presenta de modo dialógico; así, terapeuta y paciente construyen un sentido que lleva al consultante a un mayor entendimiento de 
los elementos inconscientes que se presentan en su PEV con el objetivo de que el paciente llegue a un insight o un "darse cuenta».

\section{Discusión}

En el presente apartado se discutirá la importancia que tiene el uso de las PEV como insumo y actividad en la psicoterapia dinámica y la forma en la que ésta se ve enmarcada dentro del dispositivo terapéutico.

Para Marinovic (1994), como se cita en Morales (2008), el arte permite al sujeto «integrar sentimientos contradictorios, vivencias irracionales e infantiles» (p. 23). En esta medida el autor le estaría atribuyendo al arte en sí mismo un «poder» por el cual el sujeto puede llegar a cambios profundos de su estructura psíquica. En contraposición a esto, aunque durante esta investigación se ha llegado a la conclusión de que la actividad creativa o artística per se tiene cualidades de descarga que ayudan a la contención de la ansiedad, se asienta el pensamiento de Velásquez (2010), quien sentencia que «la catarsis no es suficiente en un análisis para producir cambio psíquico» (p. 27).

Desde luego, no se está negando la importancia que tienen las creaciones artísticas, tanto como factor protector en el psiquismo de los pacientes como servicio del proceso psicoterapéutico, por lo que es indispensable advertir que la actividad creativa, desde una perspectiva de objetivos terapéuticos, tiene dos momentos o niveles.

El primer momento se refiere a la actividad creadora en sí misma. Esto se puede ver reflejado en los conceptos de sublimación - Freud-y de objeto transicional - Winnicott-, los cuales para la actual investigación constituyen un núcleo transversal: establecer un puente que comunique el mundo interno del sujeto con un principio de realidad delimitado por el mundo exterior.

Acorde con los resultados del presente estudio, la creación artística tiene por sí misma un innegable valor a nivel de regulación de la ansiedad, en la medida en que sirve como objeto transicional para evocar la presencia del terapeuta, de forma que autorregula al sujeto entre las sesiones. No obstante, cabe resaltar que dichos efectos terapéuticos son temporales y, por ende, no puede esperarse un cambio psíquico profundo. 
Reflexiones semejantes podemos hacer para el caso de la sublimación, la cual no debe ser entendida como una mera descarga energética - libido o agresión-, sino como una forma de dar resolución a una tensión inconsciente (Segal, 1991, p. 132). Sin embargo, la carga afectiva y representacional materializada en la PEV sigue permaneciendo en el plano inconsciente para el sujeto, pues según Rissetti (2011) «el arte se desenvuelve entre el mundo consciente y el inconsciente» (p. 13), y es este último fragmento, el que permanece reprimido, sobre el cual recae la tarea interpretativa del terapeuta, lo que nos lleva al siguiente nivel.

El segundo momento se establece cuando la creación del consultante es puesta en el espacio psicoterapéutico. Allí la creación es objeto de dialéctica entre psicoterapeuta y paciente a través de las técnicas verbales y la relación terapéutica. El psicoterapeuta tiene entonces la función de devolver su propia creación al paciente, así la catarsis no se presentará únicamente a nivel inconsciente, sino que trascenderá al plano de la consciencia. En definitiva, lo que en principio no puede ser tramitado por la persona en el lenguaje debido a las resistencias y los mecanismos defensivos, es exteriorizado mediante la PEV, que sigue permaneciendo como un «saber no sabido", razón por la cual es tarea de la psicoterapia que el paciente hable de ello, lo haga consciente y lo reelabore.

Por consiguiente, es sólo mediante este momento final que se hace posible promover cambios psíquicos más profundos en el paciente, como llegar a una integración del objeto y del sí mismo o constituir movimientos en rasgos de su estructura de personalidad, entre otros.

Por otro lado, para Adorna (2014), «con la escritura ganamos en libertad pues adquirimos una distancia útil que facilita poder convertirnos en los conductores y guías de nuestros pensamientos» (p. 14). Aunque la autora restringe esta cualidad a la escritura, puede ser atribuida a cualquier forma de simbolización. Esta teoría es reafirmada por Segal (1991), quien argumenta que el símbolo «representa el objeto, pero es una creación del sujeto, y en consecuencia puede utilizarse libremente» (p. 76).

Dicho de otra manera, la acción de poder crear un objeto externo con base en el mundo interno del sujeto permitiría, por un lado, que el mismo sujeto pueda «objetivarse» y, por otro, poner un tercero en la díada terapéutica. Esas 
aserciones son sostenidas por Rissetti (2011), quien respecto a la terapia de arte analítica afirma que la relación triangular en terapia es un espacio que se genera entre el consultante, el psicoterapeuta y el objeto creado (p. 14). En otras palabras, el espacio clínico se transforma en el lugar donde interactúan un yo, una otredad y un inconsciente, en este caso, en entes separados, lo que permite que los dos primeros puedan comprender y construir a través de un proceso dialógico nuevos sentidos acerca del tercero. En la figura 1 se presenta el proceso de construcción de sentido de la PEV descrito anteriormente.

Es necesario recalcar que no es el terapeuta el encargado de atribuir sentidos «objetivos» a la PEV de forma unilateral, pues como asegura Coderch (2012) acerca de la interdependencia entre la relación y la interpretación, «un proceso analítico o psicoterapéutico es una relación íntima entre dos personas y ello da lugar a que los resultados dependan de la conjunción entre estas dos mentes, únicas e irrepetibles» (p. 266).

Hay que mencionar la diferencia en el abordaje de la creación en el arte-terapia, las pruebas proyectivas y el uso de las PEV como insumo. En primer lugar, una de las capacidades básicas que debe tener un arteterapeuta es "poseer conocimientos teóricos y prácticos en artes visuales» (Asociación Profesional Española de Arteterapeutas, 2012, p. 2); dicho conocimiento es requerido, en parte, para tratar las obras de los pacientes. Por su parte, cada una de las pruebas proyectivas cuenta con un manual de interpretación para orientar la interpretación de la producción gráfica, verbal o escrita en sí misma; en el caso de la psicoterapia dinámica, y usando las PEV como insumo, las interpretaciones del psicoterapeuta deben dar relevancia, como lo establece Coderch (2012), a un componente intuitivo-empático, el cual «depende de las capacidades específicas de intuición y empatía, distintas a los conocimientos científicos, que forman parte de la personalidad de cada terapeuta» (p. 320).

Lo dicho hasta aquí supone que el componente intuitivo caracteriza el elemento subjetivo de la interpretación, sin embargo, no está desligado del razonamiento clínico que cada terapeuta ha podido construir innegablemente con base en una teoría, y más importante aún, a partir de su propia práctica clínica. Asimismo, se resalta que la interpretación —a diferencia de los test y la arteterapia - sigue teniendo un mayor énfasis en los sentidos que el paciente 
despliega en el discurso acerca de su propia PEv, que en una interpretación de la obra en sí misma, aun cuando se reconoce el conocimiento del terapeuta para interpretar cualidades objetivas de la PEV como un valor agregado, pero no es indispensable.

Figura 1. Proceso de construcción de sentido de las PEV

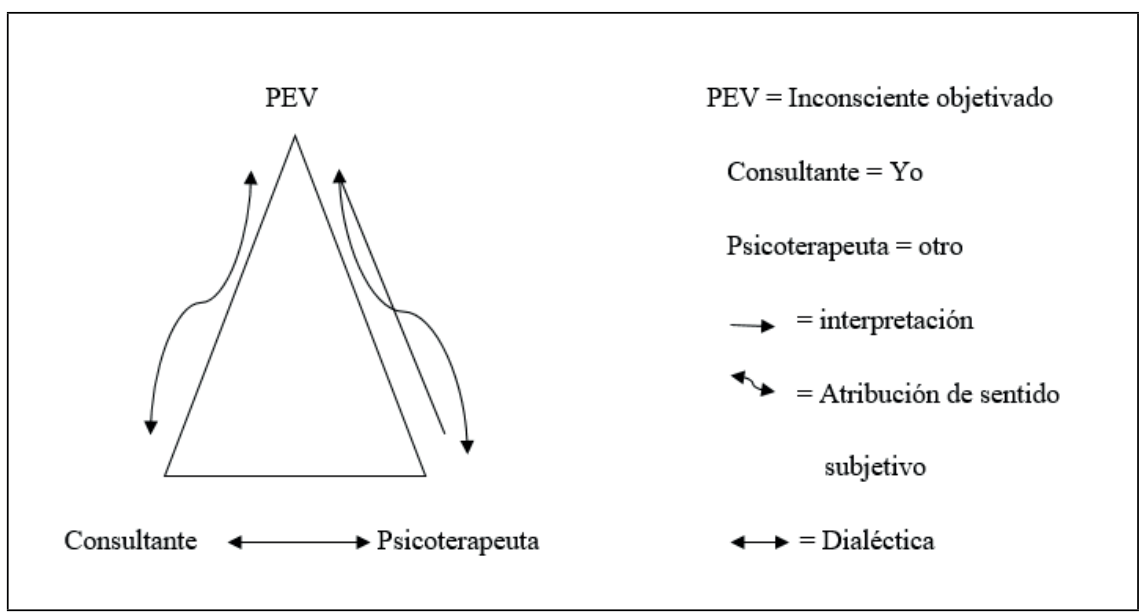

Por otra parte, los psicoterapeutas registran que durante el proceso terapéutico se van presentando cambios en las PEV de sus consultantes, lo que puede evidenciar movimientos importantes en el psiquismo de los sujetos. En esta vía se han identificado dos tipos de cambios:

a) Extensión/intensidad: en la presentación de los casos, los psicoterapeutas hacen referencia a que, durante el proceso terapéutico, algunos consultantes realizaban escritos entre sesiones para tranquilizarse, además, cuando escribían lo hacían para su terapeuta. Reportan los entrevistados que, a medida que avanza el proceso, los escritos comienzan a tener cambios, se producen con una menor frecuencia y son cada vez más cortos a tal punto que al final desaparecían.

En consonancia con lo anterior, Horner (2004) sugiere que es inevitable que durante la relación terapéutica el paciente internalice al terapeuta como objeto nuevo (p. 153); de esta forma, el consultante lo hace parte de su mundo interno, de ese modo disminuye progresivamente su necesidad de recrearlo en la produc- 
ción de un objeto externo para mitigar su ansiedad. Similarmente, Winnicott (1990) indica acerca del objeto transicional que «en un estado de buena salud el objeto no "entra", ni es forzoso que el sentimiento relacionado con él sea reprimido. No se lo olvida ni se lo llora. Pierde significación» (p. 22).

b) Forma/contenido: estos cambios en las PEV de los consultantes parecen indicar un cambio psíquico más profundo desde el punto de vista de la teoría de las relaciones objetales. Para Klein (1990), el arte tiene su explicación en el mecanismo de reparación (p. 208). Empero, hay que recordar que la reparación es el mecanismo que funciona en la posición depresiva, en la cual el sujeto ya alcanzó la integración del objeto y del sí mismo en valencias negativas y positivas.

Es por esto que Klein (1990), desde la perspectiva de Aranguren y León (2011), estaría planteando que en estructuras de menor nivel como la borderline y la psicosis, al no haber alcanzado la posición depresiva, están imposibilitadas para «la creatividad como expresión individual» (p. 452). Por el contrario, Winnicott (1984), como se cita en Aranguren y León (2011), establece que:

\begin{abstract}
A pesar de que en la psicosis exista un menoscabo en el desarrollo de la zona intermedia de la experiencia, lo cual perjudica a la creatividad [...] el individuo nace creador, y es dueño de una creatividad primaria que posee por el sólo hecho de estar vivo (p. 455).
\end{abstract}

Dicho lo anterior, se retoma un caso expuesto en las entrevistas: en él, un consultante es pintor y tiene conflictos con lo femenino. Al comienzo del proceso lleva pinturas de mujeres «demoníacas» en contextos eróticos agresivos y de trazos muy fuertes y oscuros. Observa su terapeuta que avanzado el tratamiento su paciente comienza a pintar mujeres con características más humanas, no resalta la agresividad en la sexualidad y sus trazos también comienzan a ser más suaves.

Por consiguiente, se concluye que el mecanismo de reparación no se presentó por el arte en sí mismo, ni fue condición para la creatividad del sujeto — como lo sugiere el planteamiento kleiniano-, sino que pudo darse como resultado del proceso psicoterapéutico, lo cual ayudó al sujeto a modificar sus representaciones de lo femenino, a integrar el objeto y a disminuir la severidad 
de su superyó, lo que finalmente fue proyectado y simbolizado en sus últimas pinturas. En palabras de Coderch (2006), y en referencia a la clínica kleiniana, puede decirse que en este caso el terapeuta ayudó al paciente a «transitar desde la posición esquizoparanoide a la posición depresiva» (p. 288).

En relación con la inclusión de la PEV en el proceso de terapia, algunos de los psicoterapeutas entrevistados sólo usaban dicho insumo si se presentaba de forma espontánea por parte de los consultantes. Como contraparte, otros entrevistados aludían a que, dependiendo del caso, ellos sugerían a sus pacientes que las trajesen al espacio clínico, e incluso motivaban su realización.

Probablemente el que los psicoterapeutas dinámicos no sugieran al consultante la realización de estos productos creativos, ni los solicitasen tomando una posición más activa, obedece «un celo extremo por no influir en el paciente ni alterar el "supuesto" desarrollo espontáneo de la transferencia» (Lanza, 2006, p. 158). En contraste con esto, Bleichmar, como se cita en (2015), dice que «el paciente tiene que tomar un rol activo en el proceso de su curación [...] debe ser estimulado para que el intervalo entre sesión y sesión continúe siendo un período de autoanálisis» (p. 15).

Asimismo, Fiorini (1993) llama a esta limitación en la técnica presente en diversos psicoterapeutas como una configuración mitica. Explica que la técnica psicoanalítica, la creencia en una técnica fija y universalizable es un mito y agrega que «en la práctica muchos de los autores que pretenden sustentar la "técnica" en singular con sus pacientes no pueden sostenerla y eso creo por un feliz respeto al sentido de realidad» (p. 47).

Se ha encontrado, pues, que la implementación de las Pev dentro de la psicoterapia puede hacerse de forma pasiva — partiendo de la espontaneidad del consultante - y activa — como actividad sugerida por el psicoterapeuta entre sesiones- - La utilización de dicha herramienta o insumo puede enriquecer en gran medida el espacio clínico, pudiendo darse con objetivos de encuadre, terapéuticos — primer y segundo nivel—y de evaluación, la cual no se supedita meramente a un primer momento para la identificación de conflictos, sino también en etapas más avanzadas de la psicoterapia, evidenciando los cambios que puede ir teniendo el sujeto a lo largo del proceso. 
Es menester hacer énfasis en que el uso de este insumo no tiene una estructura lineal, normativa o rígida por la cual deba regirse, ni ha de utilizarse de forma generalizable a todos los casos, pues cada psicoterapeuta puede utilizarla con propósitos distintos y de formas diferentes, dependiendo de las características de cada caso y de la subjetividad del consultante. El determinar una guía o protocolo de uso de las PEv sería caer en otras formas de utilización del arte y la creatividad en la terapia, de las cuales en el presente texto ya se ha tomado distancia y establecido una clara diferenciación.

\section{Conclusiones}

Durante la investigación se dieron encuentros y diferencias entre los psicoterapeutas respecto al uso de las PEv en el espacio clínico, esto debido a los distintos énfasis de las teorías psicoanalíticas que ellos han elegido para ejercer su práctica clínica. Sin embargo, hay una tendencia mayor a los puntos de encuentro del uso de las PEv como insumo técnico en la psicoterapia. De igual modo, es relevante resaltar que las diferencias que se encontraron no eran sustanciales, contradictorias o excluyentes, sino que se complementaban, lo que facilitó la construcción del conocimiento en este estudio.

Se puede concluir que el uso de las Pev de los consultantes en la psicoterapia dinámica ha sido un medio que ha propiciado la consecución de objetivos terapéuticos importantes. El cumplimiento de dichos objetivos no se presenta simplemente por el acto mismo de realizar un dibujo o un escrito, sino porque el uso de la PEV se ve enmarcado dentro del contexto de un dispositivo terapéutico, por lo que la PEV sirve como facilitadora del proceso clínico y como insumo significativo en la triangulación de la información constituida por los psicoterapeutas.

Aunque todos los terapeutas entrevistados habían utilizado las PEV de los consultantes en el espacio psicoterapéutico con propósito técnico —evaluativo, de intervención, informativo, relacional u otro-, ninguno lo hace bajo unos objetivos o técnicas claras, por lo que se considera que esta investigación puede servir como guía del servicio práctico de los psicoterapeutas dinámicos. 
Se concluye, entonces, que en este reporte las PEV son un insumo de gran valor para el desarrollo de la psicoterapia, pues pueden ser utilizadas por los psicoterapeutas para el cumplimiento de múltiples objetivos durante el proceso clínico, tales como: promover la asociación libre al usarla como objeto-estímulo para el despliegue del discurso y asociaciones significativas por parte del paciente; vencer resistencias en pacientes que activan defensas que pueden estancar el proceso; o cumplir una función de objeto transicional o vía sublimatoria, teniendo como resultado la contención de la ansiedad entre sesiones y su inclusión en el proceso como pago simbólico con el objetivo de afianzar el rol y el vínculo paciente-terapeuta.

Se ha podido comprender cómo las PEV se han integrado al proceso psicoterapéutico y cómo son abordadas por los psicoterapeutas a partir de técnicas como la interpretación _ la cual se presenta en forma de dialéctica para la construcción de sentidos_- además del uso de otras estrategias como los señalamientos y las preguntas exploratorias.

\section{Sugerencias}

Ya que la casuística brindada por los terapeutas se centró en estas dos formas de PEV: escritos y producciones gráficas, queda abierta la pregunta referente a otras formas de producciones que pueden presentarse en el transcurso de la psicoterapia dinámica, como la música, la danza, el teatro, la fotografía, etc.

Además surge la pregunta por otras formas de introducir los elementos estéticos como insumo en la psicoterapia dinámica y que no sean necesariamente creación del consultante — películas, series, canciones, libros, pinturas, etc.pero que de igual forma constituyan producciones con las que el paciente pueda sentirse identificado o incluso en conflicto, y a partir de los cuales pueda emerger su discurso y la creación de nuevas formas de sentido.

\section{Limitaciones}

Para comprender en mayor medida la importancia del uso de las producciones escritas y visuales de los consultantes en el espacio de la psicoterapia 
dinámica, es pertinente entrevistar no sólo a los psicoterapeutas, sino también a los pacientes para que éstos puedan dar cuenta de su percepción y sentir respecto al trabajo llevado con el psicoterapeuta que tiene como objeto mediador sus propias creaciones. Esto podría contrastarse con la información recolectada durante la investigación llevada a cabo con el propósito de dar mayor validez a los resultados.

\section{REFERENCIAS}

Abadi, S. (1996). Transiciones. Buenos Aires: Lumen.

Adorna, R. (2014). Practicando la escritura terapéutica: 79 ejercicios. Bilbao: Desclée De Bouwer.

Adorna, R., y Covarsí, J. (2016). Poesía terapéutica: 194 ejercicios para hacer un poema cada día. Bilbao: Desclée De Brouwer.

Ángel, D. (2011). Hermenéutica y métodos de investigación en ciencias sociales. Estudios Filosóficos, 44, 9-37.

Aranguren, M., y León, T. (2011). Creatividad: su expresión en la psicosis. Psicoanálisis, 33(3), 443-465.

Asociación Profesional Española de Arteterapeutas. (2012). Ejercicio del arteterapia: criterios minimos del ejercicio de la profesión. ATE. Recuperado de http://arteterapia.org.es/ejercicio-del-arteterapia/.

Bedoya, M. (2006). Acercamiento a las condiciones de posibilidad de la clinica psicológica posmoderna (Tesis de maestría). Universidad San Buenaventura, Medellín.

Coderch, J. (2012). La práctica de la psicoterapia relacional: el modelo interactivo en el campo del psicoanálisis. Madrid: Ágora Relacional.

Coderch, J. (2006). Pluralidad y diálogo en psicoanálisis. Barcelona: Herder.

Coderch, J. (1990). Teoría y técnica de la psicoterapia psicoanalítica. Barcelona: Herder.

Dumas, M., y Aranguren, M. (2013). «Beneficios del arteterapia sobre la salud mental». En: V Congreso Internacional de Investigación y Práctica Profesional en Psicología XX. Jornadas de Investigación: Noveno Encuentro de Investigadores en Psicología del Mercosur. Universidad de Buenos Aires, Buenos Aires. 
Fiorini, H. J. (1993). Estructuras y abordajes en psicoterapias psicoanaliticas. Buenos Aires: Nueva Visión.

Fiorini, H. J. (1984). Teoría y técnica de psicoterapias. Buenos Aires: Nueva Visión.

Freud, S. (1986b). Dostoievski y el parricidio. En Obras completas. Vol. xxi (pp. 171194). Buenos Aires: Amorrortu.

Freud, S. (1986c). El Moisés de Miguel Ángel. En Obras completas. Vol. XIII (pp. 213242). Buenos Aires: Amorrortu.

Freud, S. (1986d). Sobre la iniciación del tratamiento. En Obras completas. Vol. XII (pp. 121-144). Buenos Aires: Amorrortu.

Freud, S. (1986a). Un recuerdo infantil de Leonardo da Vinci. En Obras completas. Vol. XI (pp. 53-110). Buenos Aires: Amorrortu.

Hallar, R. (2015). Arte, locura y psicoterapia: una aproximación constructivista a la psicosis y a la creación artística como tratamiento. Santiago: RIL.

Horner, A. (2004). Psychoanalytic Object Relations Therapy. New York: Rowman \& Littlefield.

Klein, M. (1990). Amor, culpa y reparación: y otros trabajos (1921-1945). Buenos Aires: Paidós.

Lanza, G. (2006). El trabajo de la escritura entre sesiones. Subjetividad y procesos cognitivos, 9, 155-176.

Laplanche, J., y Pontalis, J. (1996). Diccionario de psicoanálisis. Buenos Aires: Paidós.

Martín, R. (2015). Autobiografía en psicoterapia breve: cinco horas con Mario. Revista Digital de Medicina Psicosomática y Psicoterapia, 2(5), 1-41.

Mesones, M. (2014). El "objeto transicional» en la adolescencia (Tesis de maestría). Pontificia Universidad Católica de Perú, San Miguel.

Morales, P. (2008). ¿Puede la actividad plástica ayudar en el tratamiento de personas con trastornos mentales severos?: los efectos terapéuticos del arteterapia (Tesis de pregrado). Universidad de Chile, Santiago de Chile.

Pérez, E. (2012). Técnicas de intervención dinámica y arte en pacientes con psicopatología severa. Revista de Psicología, 30(1), 129-168.

Rissetti, M. (2011). Del hacer en arte terapia: desde las emociones y en el sentir de una mujer con depresión (Tesis de pregrado). Universidad de Chile, Santiago de Chile. 
Rodríguez, D., y Niño, J. (2006). Marco comprensivo para el estudio de los procesos de formación de terapeutas. Revista Diversitas: Perspectivas en Psicología, 2(1), $42-54$.

Salamanca, A., y Martín-Crespo, C. (2007). El diseño de la investigación cualitativa. Nure Investigación, 26. Recuperado de http://www.nureinvestigacion.es/OJS/ index.php/nure/article/view/340/330

Saldaña, J. (2015). La integración resiliente en el modelo de psicoterapia breve: a propósito de la intervención terapéutica en los casos de mobbing. Revista Digital de Medicina Psicosomática y Psicoterapia, 3(5), 1-22.

Salgado, A. (2007). Investigación cualitativa: diseños, evaluación del rigor metodológico y retos. Revista Liberabit, 13, 71-78.

Segal, H. (1991). Sueño, fantasma y arte. Buenos Aires: Nueva Visión.

Shafi, N. (2010). Poetry Therapy and Schizophrenia: Clinical and Neurological Perspectives. Journal of Poetry Therapy, 23(2), 87-99.

Velásquez, D. (2010). La catarsis. El Psicoanalítico, 3, 27-31.

Winnicott, D. (1995). Realidad y juego. Buenos Aires: Gedisa.

Zurbano, A. (2007). El arte como mediador entre el artista y el trauma: acercamientos al arte desde el psicoanálisis y la escultura de Louise Bourgeois (Tesis de doctorado). Universidad del País Vasco, País Vasco. 